\title{
DIFFERENTIAL GEOMETRY OF THE COMPLEX PLANE*
}

\author{
BY \\ JULIAN LOWELL COOLIDGE
}

\section{THREADS AND CHAINS}

We shall mean by the complex plane that where $x$ and $y$ take complex as well as real values. In counting parameters, we shall always count the number of real variables involved. Thus, the plane itself is a four-parameter system, in which there are included one, two and three-parameter systems. It is assumed also that all of the functions of real parameters involved are analytic in such regions as may interest us. Finally, when we speak of a system as depending on a certain number of parameters, we mean that it depends upan that number and no less. Thus, we should not include a single point as a special case of a one-parameter system. The conjugate of any number $a$ shall be written $\bar{a}$.

A system of points depending analytically upon a single real parameter shall be called a "thread." It is conveniently represented by equations

$$
x=x(u), \quad y=y(u), \quad \bar{x}=\bar{x}(u), \quad \bar{y}=\bar{y}(u), \quad u=\bar{u} .
$$

Every thread lies upon a curve obtained by allowing $u$ to take all complex values. Two curves can not intersect in a thread, for if irreducible, they have only discreet points in common, and if reducible, they have in common either discreet points, or a common branch. Another way to indicate a thread analytically is in terms of its projections on the two axes:

$$
f(x, \bar{x})=\phi(y, \bar{y})=0 .
$$

There is one thread which is of fundamental importance in all complex geometry, namely, the "chain." This is such a system of collinear points that the cross ratio of any four is real, while the system contains the point which makes any preassigned real cross ratio with three of its members. The real domain

* Presented to the Society, December 29, 1920. 
of a real line is the standard example of a chain, and if the totality of points of a real line be represented in the Gauss plane, the points of a chain will appear as collinear or concyclic points. Two points may be connected by a simply infinite system of chains. Thus, an arbitrary chain connecting $\left(x^{\prime \prime}, y^{\prime \prime}\right)$ and $\left(x^{\prime}, y^{\prime}\right)$ may be written

$$
x=\frac{x^{\prime \prime}+t \rho x^{\prime}}{1+t \rho}, \quad y=\frac{y^{\prime \prime}+t \rho y^{\prime}}{1+t \rho}, \quad \bar{x}=\frac{\bar{x}^{\prime \prime}+t \bar{\rho} \bar{x}^{\prime}}{1+t_{\rho}}, \bar{y}=\frac{\bar{y}^{\prime \prime}+t \bar{\rho} \bar{y}^{\prime}}{1+t \bar{\rho}} .
$$

Here $t$ is the parameter that changes along the chain, while $\rho$ serves to determine which of the chains connecting the two points we have to deal with.

There is associated with each chain an involutory transformation of its line. Each point of the chain is invariant in this transformation. As for other points of the line, the relation is such that corresponding points are harmonically separated by pairs of points of the chain. In the Gauss plane this appears as a reflection in a line, or inversion in a circle. If two chains be so related to one another that the first is transformed into itself by the transformation associated with the second, then the second is anallagmatic in the transformation associated with the first. We shall call two such chains "orthogonal." They will appear in the Gauss plane as mutually perpendicular circles or lines.

\section{Congruences AND CURVES}

A system of points which depends upon two parameters shall be called a "congruence." The real domain furnishes one example of such a variety; a curve furnishes another such. The fundamental question connected with any congruence is this: is it, or is it not, a curve? Let the congruence be represented by the equations

$$
x=x(u, v), \quad y=y(u, v), \bar{x}=\bar{x}(u, v), \bar{y}=\bar{y}(u, v), \quad u=\bar{u}, v=\bar{v}
$$

If this be a curve, then $\frac{d y}{d x}$ must be independent of $\frac{d v}{d u}$, which involves the condition

$$
\frac{\partial(x, y)}{\partial(u, v)} \equiv 0
$$

Conversely, if this equation holds for all real values of $u, v$, since only analytic functions are involved, it holds for all values, real or complex. Hence there is a functional relation between $x$ and $y$, or we have a curve. 
THEOREM 1. A necessary and sufficient condition that the congruence (4) should be a curve is that the equation (5) should hold for all real values of the parameters $u$ and $v .^{*}$

If the congruence be given by two equations

$$
F(x, y, \bar{x}, \bar{y})=\bar{F}(\bar{x}, \bar{y}, x, y)=0,
$$

it will represent a curve when, and only when, it is possible to eliminate $d \bar{x}$ and $d \bar{y}$ from the differentials of these expressions, i.e.

$$
\frac{\partial(F, \bar{F})}{\partial(x, y)}=\frac{\partial(\bar{F}, F)}{\partial(\bar{x}, \bar{y})}=0,
$$

for all points of the congruence.

Suppose that our congruence is not a curve. A point thereof where equation (5) is not satisfied shall be called a "usual" point. The equation of the tangent at such a point will be

$$
\left[(\xi-x) \frac{\partial x}{\partial u}+(\eta-y) \frac{\partial y}{\partial u}\right] d u+\left[(\xi-x) \frac{\partial x}{\partial v}+(\eta-y) \frac{\partial y}{\partial v}\right] d v=0
$$

This system of concurrent lines depends linearly on the real homogeneous parameters $d u, d v$. It will clearly cut an arbitrary transversal in a chain of points, and so may properly be called a "chain" of lines.

THEOREM 2. If a congruence be not a curve, the tangents at a usual point will generate a chain of lines.

We must now make a short digression into the theory of transformations of the plane. If $(x, y)$ and $\left(x^{\prime}, y^{\prime}\right)$ be corresponding points in a transformation, we may either express the coördinates of the latter in terms of the former, or express both in terms of auxiliary variables $u, v$. The transformation is directly conformal, if

$$
\frac{\partial\left(x, x^{\prime}\right)}{\partial(u, v)}=\frac{\partial\left(y, y^{\prime}\right)}{\partial(u, v)} ; \quad \frac{\partial\left(x, y^{\prime}\right)}{\partial(u, v)}=-\frac{\partial\left(y, x^{\prime}\right)}{\partial(u, v)} .
$$

It is inversely conformal, if

$$
\frac{\partial\left(x, x^{\prime}\right)}{\partial(u, v)}=-\frac{\partial\left(y, y^{\prime}\right)}{\partial(u, v)} ; \quad \frac{\partial\left(x, y^{\prime}\right)}{\partial(u, v)}=\frac{\partial\left(y, x^{\prime}\right)}{\partial(u, v)},
$$

* It is not perfectly clear to whom the credit for this theorem belongs. Study, Ausgewählte Gegenstände der Geometrue, Berlin, 1911, p. 43, ascribes it to the second section of Segre's Nuovo campo di ricerche geometriche, A t $\mathrm{i}$ de 11 a $\mathrm{R}$ e a 1 e $\mathrm{Acc}$ a d e m i a de 11 e S c i e n z e d i T o r i n o, vol. 25 (1889-90), but only the necessity of the condition seems to be there proved. However, it is certain that Segre was aware of the sufficiency also. Our Theorem 2 is found on p. 437. 
as these reduce to the Cauchy-Riemann equations when $x=u, y=v$.

It is directly equi-areal, if

$$
\frac{\partial(x, y)}{\partial(u, v)}=\frac{\partial\left(x^{\prime}, y^{\prime}\right)}{\partial(u, v)}
$$

It is inversely equi-areal, if

$$
\frac{\partial(x, y)}{\partial(u, v)}=-\frac{\partial\left(x^{\prime}, y^{\prime}\right)}{\partial(u, v)}
$$

These six jacobians are connected by the identical relation

$$
\frac{\partial(x, y)}{\partial(u, v)} \frac{\partial\left(x^{\prime}, y^{\prime}\right)}{\partial(u, v)}=\frac{\partial\left(x, x^{\prime}\right)}{\partial(u, v)} \frac{\partial\left(y, y^{\prime}\right)}{\partial(u, v)}-\frac{\partial\left(x, y^{\prime}\right)}{\partial(u, v)} \frac{\partial\left(y, x^{\prime}\right)}{\partial(u, v)} .
$$

\section{LAGUERRE REPRESENTATION; INVARIANTS}

In studying a congruence of points, there is a very great advantage in keeping in mind the method of representing a complex point devised by Laguerre.* This consists essentially in representing each complex point by the ordered pair of real points at a zero distance therefrom, the conjugate imaginary point being thus represented by the same real pair in reverse order. The representation works without hitch for all finite points. Analytically we have

$$
\begin{aligned}
& x=\frac{1}{2}\left(X+X^{\prime}\right)+\frac{i}{2}\left(Y-Y^{\prime}\right), \quad y=\frac{1}{2}\left(Y+Y^{\prime}\right)-\frac{i}{2}\left(X-X^{\prime}\right) \\
& \bar{x}=\frac{1}{2}\left(X+X^{\prime}\right)-\frac{i}{2}\left(Y-Y^{\prime}\right), \quad \bar{y}=\frac{1}{2}\left(Y+Y^{\prime}\right)+\frac{i}{2}\left(X-X^{\prime}\right) .
\end{aligned}
$$

There are certain invariants connected with our congruence which are of fundamental importance; they are

$$
\begin{gathered}
J+i J^{\prime}=2 \frac{\partial(x, y)}{\partial(u, v)} ; \quad J-i J^{\prime}=2 \frac{(\partial \bar{x}, \bar{y})}{\partial u, v)} \\
i H=\frac{\partial(x, \bar{x})}{\partial(u, v)}+\frac{\partial(y, \bar{y})}{\partial(u, v)} ; \quad K=\frac{\partial(x, \bar{y})}{\partial(u, v)}-\frac{\partial(y, \bar{x})}{\partial(u, v)}
\end{gathered}
$$

Each of these expressions is unaltered in absolute value by a real congruent transformation of the plane, and is merely multiplied by the jacobian if we change real parameters. If we express these invariants in terms of the Laguerre representation, we have

* Sur l'emploi des imaginaires dans la géométrie, Collected Works, vol. 2 (1905), pp. 88, 98. 


$$
\begin{gathered}
{\left[J+i J^{\prime}\right]=\left[\frac{\partial\left(X, Y^{\prime}\right)}{\partial(u, v)}-\frac{\partial\left(Y, X^{\prime}\right)}{\partial(u, v)}\right]+i\left[\frac{\partial\left(X, X^{\prime}\right)}{\partial(u, v)}+\frac{\partial\left(Y, Y^{\prime}\right)}{\partial(u, v)}\right]} \\
{\left[J-i J^{\prime}\right]=\left[\frac{\partial\left(X, Y^{\prime}\right)}{\partial(u, v)}-\frac{\partial\left(Y, X^{\prime}\right)}{\partial(u, v)}\right]-i\left[\frac{\partial\left(X, X^{\prime}\right)}{\partial(u, v)}+\frac{\partial\left(Y, Y^{\prime}\right)}{\partial(u, v)}\right]} \\
H=\left[\frac{\left(\partial X^{\prime}, Y^{\prime}\right)}{\partial(u, v)}-\frac{\partial(X, Y)}{\partial(u, v)}\right] \\
K=\left[\frac{\partial\left(X^{\prime}, Y^{\prime}\right)}{\partial(u, v)}+\frac{\partial(X, Y)}{\partial(u, v)}\right]
\end{gathered}
$$

We find by using (7) twice

$$
\begin{gathered}
{\left[\frac{\partial(x, \bar{x})}{\partial(u, v)}-\frac{\partial(y, \bar{y})}{\partial(u, v)}\right]^{2}+\left[\frac{\partial(x, \bar{y})}{\partial(u, v)}+\frac{\partial(y, \bar{x})}{\partial(u, v)}\right]^{2}=-H^{2}+K^{2}-J^{2}-J^{\prime 2}} \\
=-\left\{\left[\frac{\partial\left(X, X^{\prime}\right)}{\partial(u, v)}-\frac{\partial\left(Y, Y^{\prime}\right)}{\partial(u, v)}\right]^{2}+\left[\frac{\partial\left(X, Y^{\prime}\right)}{\partial(u, v)}+\frac{\partial\left(Y, X^{\prime}\right)}{\partial(u, v)}\right]^{2}\right\}
\end{gathered}
$$

\section{Conformal AND EQUi-AREAL RELATIONS}

We have now finished the dreary task of formula grinding, at least for the moment; let us draw some conclusions. Suppose that we have a curve in the complex plane. That by Theorem 1 , and by Formula $B$, will give an inversely conformal transformation of the Laguerre representatives, unless

$$
\frac{\partial(X, Y)}{\partial(u, v)} \frac{\partial\left(X^{\prime}, Y^{\prime}\right)}{\partial(u, v)}=0
$$

If the first factor vanish, then either $(X, Y)$ is stationary, or moves along a curve. Making the second assumption we may take $u$ as the single real parameter on which $x$ and $y$ depend; then $\frac{\partial X}{\partial v}=\frac{\partial Y}{\partial v}=0$, and either $\frac{\partial X^{\prime}}{\partial v}=\frac{\partial Y^{\prime}}{\partial v}=0$, which is inadmissible, or $\left(\frac{\partial(X)}{\partial u}\right)^{2}+\left(\frac{\partial Y}{\partial u}\right)^{2}=0$, i. e., $\frac{\partial X}{\partial u}=\frac{\partial Y}{\partial u}=0$ and $(X$, ( $Y$ is fiixed. When $(X, Y)$ is fixed, the curve traced by $(x, y)$ is a minimal line.

THEOREM 3. If a congruence consist in a curve, not a minimal line, the corresponding relation between the Laguerre representatives is inversely conformal.*

* Study, loc. cit., p. 63. This author studies not only the Laguerre representation but also another closely allied, where a complex curve appears as a special type of directly equi-areal transformations. 
We have covered the case of a congruence where the invariant $J+i J^{\prime}$ is zero. Suppose next that

$$
H=K=0 \text {. }
$$

We have at once from (9)

THEOREM 4. If a congruence be inversely conformally related to its conjugate, the Laguerre representatives will trace two curves.

This theorem might also have been predicted from the fact that we pass from a pair of conjugate imaginary points to their Laguerre representatives by a transformation of period two. Turning next to equation (10) we see that the left hand side can vanish only when each of the individual squares does, and the same happens on the right.

THEOREM 5. A necessary and sufficient condition that the relation between a congruence, not a curve, and its conjugate should be directly conformal, is that the same should be true of the Laguerre representatives.

THEOREM 6. If the relation between a congruence and its conjugate be not inversely conformal yet the equation

$$
\mathrm{H}=0
$$

subsist, then the corresponding Laguerre representatives are connected by a directly equi-areal relation.

THEOREM 7. If the relation between a congruence and its conjugate be not inversely conformal, yet the equation

$$
K=0
$$

subsists, then the corresponding Laguerre representatives are connected by an inversely equi-areal transformation.

The congruences where $(x, y)$ and $(\bar{x}, \bar{y})$ trace curves are a special case of those where points invariantly connected with them do so. We mean by invariantly connected points these whose distances from $(x, y)$ and $(\bar{x}, \bar{y})$ bear given ratios to the distances of these points from one another. It is easy to show that the general expression for the coördinates of such a point is

$$
x^{\prime}=\frac{x-r \bar{x}}{1-r}-\rho \frac{y-\bar{y}}{1-r}, \quad y^{\prime}=\frac{\rho(x-\bar{x})}{1-r}+\frac{y-r \bar{y}}{1-r} .
$$

We find, with a little patience, that

$$
\frac{\partial\left(x^{\prime}, y^{\prime}\right)}{\partial(u, v)}=\alpha\left[J+i J^{\prime}\right]+\beta\left[J-i J^{\prime}\right]+\gamma H+\delta K,
$$


where

$$
\alpha=\frac{1+\rho^{2}}{2}, \beta=\frac{r^{2}+\rho^{2}}{2}, \gamma=i \rho(r-1), \delta=-\left(r+\rho^{2}\right),
$$

with the identical relation

$$
\delta^{2}-\gamma^{2}=4 \alpha \beta
$$

The equation $\frac{\partial\left(x^{\prime}, y^{\prime}\right)}{\partial(u, v)}=0$ will involve

$$
\frac{\partial\left(\bar{x}^{\prime}, \bar{y}^{\prime}\right)}{\partial(u, v)}=0
$$

so that

$$
\bar{\alpha}\left[J-i J^{\prime}\right]+\bar{\beta}\left[J+i J^{\prime}\right]+\bar{\gamma} H+\bar{\delta} K=0 .
$$

The two conditions are equivalent if

$$
\alpha: \bar{\beta}=\beta: \bar{\alpha}=\gamma: \bar{\gamma}=\delta: \bar{\delta} .
$$

When $\rho \neq 0$, this involves $r=\rho / \bar{\rho}$.

The most interesting case is where $\left(x^{\prime}, y^{\prime}\right)$ is in the line from $(x, y)$ to $(\bar{x}, \bar{y})$. Here $\rho=0$.

$$
\begin{aligned}
& \left(J+i J^{\prime}\right)+r^{2}\left(J-i J^{\prime}\right)-2 r K=0, \\
& \left(J-i J^{\prime}\right)+\bar{r}^{2}\left(J+i J^{\prime}\right)-2 \bar{r} K=0, \\
& \quad r(r \bar{r}-1)\left(J-i J^{\prime}\right)=\bar{r}(r \bar{r}-1)\left(J+i J^{\prime}\right) .
\end{aligned}
$$

If

$$
r \bar{r}-1=0,
$$

the point $\left(x^{\prime}, y^{\prime}\right)$ is real; the two equations (11) amount to a single equation. An interesting special case is where

$$
J+i J^{\prime}=J-i J^{\prime}, \quad K=0 .
$$

Here the two points on the line from $(x, y)$ to $(\bar{x}, \bar{y})$ which form a square with the Laguerre representatives trace two lines. These points are also used to represent the given complex points. If the equation above connecting $r$ and $\bar{r}$ be not fulfilled, we have

$$
\frac{\partial(x, y)}{\partial(u, v)}=\frac{r}{\bar{r}} \frac{\partial(\bar{x}, \bar{y})}{\partial(u, v)}
$$

THEOREM 8. If two conjugate imaginary points trace such congruences that a not real point which divides their segment in a constant ratio traces a curve, then corresponding areas traced in the two congruences bear to one another a fixed ratio. 


\section{CONGRUENCES WHICH INCLUDE THREADS OF GIVEN TYPE}

Let us find out under what circumstances a congruence will include a set of threads lying on minimal lines. As $(x, y)$ or $(\bar{x}, \bar{y})$ traces such a thread, one I,aguerre representative must remain fixed, or

$$
\begin{gathered}
\frac{\partial(X, Y)}{\partial(u, v)} \frac{\partial\left(\bar{X}^{\prime} \bar{Y}^{\prime}\right)}{\partial(u, v)}=0, \\
K^{2}-H^{2}=0 .
\end{gathered}
$$

We pass to the more difficult question of finding the necessary and sufficient condition that there should be a normal net in our congruence, i. e., two sets of threads cutting at right angles at each point, either of the whole congruence, or of a certain two-dimensional region thereof. We must have two consistent. equations.

$$
\begin{aligned}
& d x \delta x+d y \delta y=0 \\
& d \bar{x} \delta \bar{x}+d \bar{y} \delta \bar{y}=0 .
\end{aligned}
$$

In the Laguerre representation we have

$$
\begin{aligned}
& d X \delta X^{\prime}+\delta X d X^{\prime}+d Y \delta Y^{\prime}+\delta Y d Y^{\prime}=0 \\
& d X^{\prime} \delta Y^{\prime}-d X \delta Y^{\prime}+\delta X^{\prime} d Y-\delta X d Y^{\prime}=0
\end{aligned}
$$

Let us choose $X$ and $Y$ as parameters $u$ and $v$;

$$
\begin{array}{r}
d X\left[\frac{\partial X^{\prime}}{\partial X} \delta X+\frac{\partial X^{\prime}}{\partial Y} \delta Y\right]+\delta X\left[\frac{\partial X^{\prime}}{\partial X} d X+\frac{\partial X^{\prime}}{\partial Y} d Y\right]+d Y\left[\frac{\partial Y^{\prime}}{\partial X} \delta X+\frac{\partial Y^{\prime}}{\partial Y} \delta Y\right] \\
+\delta Y\left[\frac{\partial Y^{\prime}}{\partial X} d X+\frac{\partial Y^{\prime}}{\partial Y} d Y\right]=0 \\
\delta Y\left[\frac{\partial X^{\prime}}{\partial X} d X+\frac{\partial X^{\prime}}{\partial Y} d Y\right]-d X\left[\frac{\partial Y^{\prime}}{\partial X} \delta X+\frac{\partial Y^{\prime}}{\partial Y} \delta Y\right]+d Y\left[\frac{\partial^{\prime} X}{\partial X} \delta X+\frac{\partial X^{\prime}}{\partial Y} \delta Y\right] \\
-\delta X\left[\frac{\partial Y^{\prime}}{\partial X} d X+\frac{\partial Y^{\prime}}{\partial Y} d Y\right]=0 .
\end{array}
$$

Eliminating $\delta X$ and $\delta Y$

$$
\begin{array}{r}
{\left[\left(\frac{\partial X^{\prime}}{\partial X}\right)^{2}+\left(\frac{\partial Y^{\prime}}{\partial X}\right)^{2}-\left(\frac{\partial\left(X^{\prime}, Y^{\prime}\right)}{\partial(X, Y)}\right)^{2}\right] d X^{2}+2\left[\frac{\partial X^{\prime}}{\partial X} \frac{\partial X^{\prime}}{\partial Y}+\frac{\partial Y^{\prime}}{\partial X} \frac{\partial Y^{\prime}}{\partial Y}\right] d X d Y} \\
+\left[\left(\frac{\partial X^{\prime}}{\partial Y}\right)^{2}+\left(\frac{\partial Y^{\prime}}{\partial Y}\right)^{2}-\frac{\partial\left(X^{\prime}, Y^{\prime}\right)}{\partial(X, Y)}\right] d Y^{2}=0 .
\end{array}
$$

The condition for real roots is

$$
\frac{\partial\left(X^{\prime}, Y^{\prime}\right)}{\partial(X, Y)}\left[\left(\frac{\partial X^{\prime}}{\partial X}\right)^{2}+\left(\frac{\partial X^{\prime}}{\partial Y}\right)^{2}+\left(\frac{\partial Y^{\prime}}{\partial X}\right)^{2}+\left(\frac{\partial Y^{\prime}}{\partial Y}\right)^{2}-2 \frac{\partial\left(X^{\prime}, Y^{\prime}\right)}{\partial(X, Y)}\right]>0
$$


The second factor is the sum of the squares of two binomials, and will be equal to zero only when the relation between $(X, Y)$ and $\left(X^{\prime}, Y^{\prime}\right)$, or that between $(x, y)$ and $(\bar{x}, \bar{y})$, is directly conformal. As for the first factor, since

$$
\frac{\partial\left(X^{\prime}, Y^{\prime}\right)}{\partial(u, v)}=\frac{\partial\left(X^{\prime}, Y^{\prime}\right)}{\partial(X, Y)} \frac{\partial(X, Y)}{\partial(u, v)}
$$

we may write our condition

$$
\begin{gathered}
\frac{\partial(X, Y)}{\partial(u, v)} \frac{\partial\left(X^{\prime}, Y^{\prime}\right)}{\partial(u, v)}>0, \\
K^{2}-H^{2}>0 .
\end{gathered}
$$

The next to last inequality shows that in the Laguerre representation, corresponding infinitesimal triangles have the same sign, that is to say, corresponding differentials rotate in the same direction.

THEOREM 9. A necessary and sufficient condition that a congruence, not a curve, should contain an orthogonal net is that the relation with the conjugate congruence should not be conformal, and that in the Laguerre representation corresponding rotations about corresponding points go in the same sense.

THEOREM 10. A congruence which contains a set of minimal threads, or is conformally related to the conjugate, can not contain one and only one normal net.

Let us next see under what circumstances a congruence can contain a thread whose tangents are parallel to the corresponding tangents in the conjugate congruence. We have here

$$
\begin{gathered}
d x d \bar{y}-d y d \bar{x}=0, \\
\left(\frac{\partial x}{\partial u} \frac{\partial \bar{y}}{\partial u}-\frac{\partial y}{\partial u} \frac{\partial \bar{x}}{\partial u}\right) d u^{2}+\left(\frac{\partial x}{\partial u} \frac{\partial \bar{y}}{\partial v}+\frac{\partial x}{\partial v} \frac{\partial \bar{y}}{\partial u}-\frac{\partial y}{\partial u} \frac{\partial \bar{x}}{\partial v}-\frac{\partial y}{\partial v} \frac{\partial \bar{x}}{\partial u}\right) d u d v \\
+\left[\frac{\partial x}{\partial v} \frac{\partial \bar{y}}{\partial v}-\frac{\partial y}{\partial v} \frac{\partial \bar{x}}{\partial v}\right]^{2} d v^{2}=0 \\
{\left[\left(\frac{\partial(x, \bar{y})}{\partial(u, v)}\right)^{2}+\left(\frac{\partial(y, \bar{x})}{\partial(u, v)}\right)\right]^{2}-2\left[\frac{\partial(x, \bar{x})}{\partial(u, v)} \frac{\partial(y, \bar{y})}{\partial(u, v)}+\frac{\partial(x, \bar{y})}{\partial(u, v)} \frac{\partial(y, \bar{x})}{\partial(u, v)}\right] \leqq 0}
\end{gathered}
$$

Hence by (7),

THEOREM 11. A necessary and sufficient condition that a congruence should contain a system of threads where a point moves parallel to its conjugate is

$$
H^{2}-J^{2}-J^{\prime 2} \leqq 0
$$

The method employed to reach (9) will yield, with much less trouble, 
THEOREM 12. A neccesary and sufficient condition that a congruence should be such that in the Laguerre representation there is a system of curves parallel to their corresponding curves is

$$
J^{2}-\left(K^{2}-H^{2}\right) \geqq 0 .
$$

THEOREM 13. A necessary and sufficient condition that a congruence should be such that in the Laguerre representation a system of curves can be found whose tangents are perpendicular to the corresponding tangents to the corresponding curves is

$$
J^{\prime 2}-\left(K^{2}-H^{2}\right) \geqq 0 \text {. }
$$

THEOREM 14. If a congruence, not a curve, have a set of threads along which a point moves parallel to its conjugate, then in the Laguerre representation there is a system of curves parallel to those to which they correspond.

Let us pass to certain slightly different considerations. Is it possible that the distance between two points of a congruence should be independent of the path? This certainly is the case if the congruence be a curve. On the other hand, when this condition is fulfilled, the distance is a function of the end-points, only, and the squared distance element

$$
d s^{2}=E d u^{2}+2 F d u d v+G d v^{2}
$$

must be the square of an exact differential, or

$$
\left[\frac{\partial(x, y)}{\partial(u, v)}\right]^{2}=0 .
$$

THEOREM 15. The only congruences where the distances of points are independent of the paths are curves.

\section{TANGENT LINES AND ChAINS}

We saw in Theorem 2 that the tangents at a usual point of a congruence which is not a curve will generate a chain of lines. It is time to look more closely into this figure. Let the equations of the congruence be expressed in power series:

$$
\begin{aligned}
& x=x(u, v)+(-u) \frac{\partial x}{\partial u}+(V-v) \frac{\partial x}{\partial v}+\frac{1}{2}\left[(U-u)^{2} \frac{\partial^{2} x}{\partial u^{2}}\right. \\
&\left.+2(U-u)(V-v) \frac{\partial^{2} x}{\partial u \partial v}+(V-v)^{2} \frac{\partial^{2} x}{\partial v^{2}}\right]+\cdots, \\
& y=y(u, v)+(U-u) \frac{\partial y}{\partial u}+(V-v) \frac{\partial y}{\partial v}+\frac{1}{2}\left[(U-u)^{2} \frac{\partial^{2} y}{\partial u^{2}}\right. \\
&\left.+2(U-u)(V-v) \frac{\partial^{2} y}{\partial u \partial v}+(V-v)^{2} \frac{\partial^{2} y}{\partial v^{2}}\right]+\cdots
\end{aligned}
$$


Let us find the intersection with $y^{\prime}=y(u, v)+\lambda\left[x^{\prime}-x(u, v)\right]$;

$$
\begin{aligned}
0= & {\left[\frac{\partial y}{\partial u}-\lambda \frac{\partial x}{\partial u}\right](U-u)+\left[\frac{\partial y}{\partial v}-\lambda \frac{\partial x}{\partial v}\right](V-v) } \\
+ & \frac{1}{2}\left\{\left[\frac{\partial^{2} y}{\partial u^{2}}-\lambda \frac{\partial^{2} x}{\partial u^{2}}\right](U-u)^{2}+2\left[\frac{\partial^{2} y}{\partial u \partial v}-\lambda \frac{\partial^{2} x}{\partial u \partial v}\right](V-u)(V-v)\right. \\
& \left.+\left[\frac{\partial^{2} y}{\partial v^{2}}-\lambda \frac{\partial^{2} x}{\partial v^{2}}\right](V-v)^{2}\right\}+\cdots, \\
0= & {\left[\frac{\partial \bar{y}}{\partial u}-\bar{\lambda} \frac{\partial \bar{x}}{\partial u}\right](U-u)+\left[\frac{\partial \bar{y}}{\partial v}-\bar{\lambda} \frac{\partial \bar{x}}{\partial v}\right](V-v) } \\
& +\frac{1}{2}\left\{\left[\frac{\partial^{2} \bar{y}}{\partial u^{2}}-\bar{\lambda} \frac{\partial^{2} \bar{x}}{\partial u^{2}}\right](U-u)^{2}+2\left[\frac{\partial^{2} \bar{y}}{\partial u \partial v}-\bar{\lambda} \frac{\partial^{2} \bar{x}}{\partial u \partial v}\right] d u d v\right. \\
& \left.+\left[\frac{\partial^{2} \bar{y}}{\partial v^{2}}-\bar{\lambda} \frac{\partial^{2} \bar{x}}{\partial v^{2}}\right](V-v)^{2}\right\}+\cdots .
\end{aligned}
$$

We have two curves in the $(U, V)$ plane, meeting at $(u, v)$. They will be tangent there if

$$
\begin{gathered}
\left|\begin{array}{l}
\frac{\partial \bar{y}}{\partial u}-\bar{\lambda} \frac{\partial \bar{x}}{\partial u} \frac{\partial \bar{y}}{\partial v}-\bar{\lambda} \frac{\partial \bar{x}}{\partial v} \\
\frac{\partial y}{\partial u}-\lambda \frac{\partial x}{\partial u} \frac{\partial y}{\partial v}-\lambda \frac{\partial x}{\partial v}
\end{array}\right|=0, \\
\lambda \bar{\lambda} \frac{\partial(x, \bar{x})}{\partial(u, v)}-\lambda \frac{\partial(x, \bar{y})}{\partial(u, v)}-\bar{\lambda} \frac{\partial(y, \bar{x})}{\partial(u, v)}+\frac{\partial(y, \bar{y})}{\partial(u, v)}=0 .
\end{gathered}
$$

This equation is unaltered when we replace $\lambda, \bar{\lambda}$ by their conjugates $\bar{\lambda}, \lambda$ if, and only if

$$
K=0 \text {. }
$$

It is unaltered when we replace $\lambda$ and $\bar{\lambda}$ by their diametral values $-1 / \bar{\lambda},-1 / \lambda$ if and only if

$$
H=0 .
$$

THEOREM 16. A necessary and sufficient condition that the tangents at an arbitrary usual point of a congruence, not a curve, should have, in pairs, conjugate imaginary slopes, is that the corresponding transformation in the Laguerre representations should be inversely equi-areal; the slopes will be diametral imaginaries. in pairs when the transformation is directly equi-areal.

Let us see whether we can find any osculating tangents which have threepoint contact with the congruence: In the $(U, V)$ plane we must have two curves which not only touch, but have the same curvature. 
Let the curves be $F(U, V)=\bar{F}(U, V)=0$;

$$
\begin{gathered}
\frac{\partial(F, \bar{F})}{\partial(U, V)}=0, \quad U=u, \quad V=v \\
\frac{\frac{\partial^{2} F}{\partial u^{2}}\left(\frac{\partial F}{\partial v}\right)^{2}+\frac{\partial^{2} F}{\partial v^{2}}\left(\frac{\partial F}{\partial u}\right)^{2}-2 \frac{\partial^{2} F}{\partial u \partial v} \frac{\partial F}{\partial u} \frac{\partial F}{\partial v}}{\left[\left(\frac{\partial F}{\partial u}\right)^{2}+\left(\frac{\partial F}{\partial v}\right)^{2}\right]^{3 / 2}} \\
=\frac{\frac{\partial^{2} \bar{F}}{\partial u^{2}}\left(\frac{\partial \bar{F}}{\partial v}\right)^{2}+\frac{\partial^{2} \bar{F}}{\partial v^{2}}\left(\frac{\partial \bar{F}}{\partial u}\right)^{2}-2 \frac{\partial^{2} \bar{F}}{\partial u \partial v} \frac{\partial \bar{F}}{\partial u} \frac{\partial \bar{F}}{\partial v}}{\left[\left(\frac{\partial \bar{F}}{\partial u}\right)^{2}+\left(\frac{\partial \bar{F}}{\partial v}\right)^{2}\right]^{3 / 2}}
\end{gathered}
$$

For this purpose it is necessary and sufficient that the following expression should be equal to its conjugate:

$$
\begin{aligned}
& \left(\frac{\partial^{2} y}{\partial u^{2}}-\lambda \frac{\partial^{2} x}{\partial u^{2}}\right)\left(\frac{\partial y}{\partial v}-\lambda \frac{\partial x}{\partial v}\right)^{2}+\left(\frac{\partial^{2} y}{\partial v^{2}}-\lambda \frac{\partial^{2} x}{\partial v^{2}}\right)\left(\frac{\partial y}{\partial v}-\lambda \frac{\partial x}{\partial v}\right)^{2} \\
& -2\left(\frac{\partial^{2} y}{\partial u \partial v}-\lambda \frac{\partial^{2} x}{\partial u \partial v}\right)\left(\frac{\partial y}{\partial u}-\lambda \frac{\partial x}{\partial u}\right)\left(\frac{\partial y}{\partial v}-\lambda \frac{\partial x}{\partial v}\right) \\
& {\left[\left(\frac{\partial y}{\partial u}-\lambda \frac{\partial x}{\partial u}\right)^{2}+\left(\frac{\partial y}{\partial v}-\lambda \frac{\partial x}{\partial v}\right)^{2}\right]^{3 / 2}}
\end{aligned}
$$

The general solution of (15) is

$$
\begin{aligned}
& \lambda=\frac{\frac{\partial y}{\partial u}+X \frac{\partial y}{\partial v}}{\frac{\partial x}{\partial u}+X \frac{\partial x}{\partial v}}, \quad X=\frac{\frac{\partial y}{\partial u}-\lambda \frac{\partial x}{\partial u}}{\frac{\partial y}{\partial v}-\lambda \frac{\partial x}{\partial v}} \\
& \frac{\partial y}{\partial u}-\lambda \frac{\partial x}{\partial u}=X\left(\frac{\partial y}{\partial v}-\lambda \frac{\partial x}{\partial v}\right)=\frac{X \frac{\partial(x, y)}{\partial(u, v)}}{\frac{\partial x}{\partial u}+X \frac{\partial x}{\partial v}} \\
& \frac{\left(\frac{\partial^{2} y}{\partial u^{2}}-\lambda \frac{\partial^{2} x}{\partial u^{2}}\right)-2 X\left(\frac{\partial^{2} y}{\partial u \partial v}-\lambda \frac{\partial^{2} x}{\partial u \partial v}\right)+X^{2}\left(\frac{\partial^{2} y}{\partial v^{2}}-\lambda \frac{\partial^{2} x}{\partial v^{2}}\right)}{\left(\frac{\partial y}{\partial v}-\lambda \frac{\partial x}{\partial v}\right)\left(1+X^{2}\right)^{3 / 2}} \\
& =\frac{\left(\frac{\partial^{2} \bar{y}}{\partial u^{2}}-\bar{\lambda} \frac{\partial^{2} \bar{x}}{\partial u^{2}}\right)-2 X\left(\frac{\partial^{2} \bar{y}}{\partial u \partial v}-\bar{\lambda} \frac{\partial^{2} \bar{x}}{\partial u \partial v}\right)+X^{2}\left(\frac{\partial^{2} \bar{y}}{\partial v^{2}}-\bar{\lambda} \frac{\partial^{2} \bar{x}}{\partial v^{2}}\right)}{\left(\frac{\partial \bar{y}}{\partial v}-\bar{\lambda} \frac{\partial \bar{x}}{\partial v}\right)\left(1+X^{2}\right)^{3 / 2}} .
\end{aligned}
$$


Substituting the values of $\lambda$ and $\bar{\lambda}$, we get the real equation

$$
P X^{3}+Q X^{2}+R X+S=0 \text {. }
$$

This equation will surely have one real root, perhaps three. Hence, at a usual point of such a congruence there is surely one osculating tangent, and there may be three of them. A thread whose tangents osculate the congruence shall be called "asymptotic:"

THEOREM 17. Every congruence, not a curve, contains a system of asymptotic threads of which at least one passes through every usual point.

There remain the unusual points. These are characterized by the equations

$$
\frac{(\partial x, y)}{\partial(u, v)}=\frac{(\partial \bar{x}, \bar{y})}{\partial(u, v)}=0 .
$$

Usually these equations are distinct. If, however, they are identical, there is a whole thread of unusual points.

THEOREM 18. A necessary and sufficient condition that there should be a thread of unusual points is that in the Laguerre representation there should be a curve of points where the transformation is inversely conformal.

\section{THREE-PARAMETER SYSTEMS}

There are two natural analytic methods for representing three-parameter point systems, namely

$$
\begin{gathered}
x=x(u, v, w), \quad y=y(u, v, w), \bar{x}=\bar{x}(u, v, w), \\
\bar{y}=\bar{y}(u, v, w), u=\bar{u}, v=\bar{v}, w=\bar{w} \\
F(x, y, \bar{x}, \bar{y})=0 .
\end{gathered}
$$

The fundamental question to be asked about any such system is whether it does or does not contain completely a curve. A chain of lines gives an example of one sort of system, the totality of points such that the sum of the squares of the absolute values of their distances from the axes has an absolute value unity gives an example of the other. Let us find a necessary and sufficient condition that our system (16) should contain a curve. The curve shall be characterized by the equation

$$
\phi(u, v, w)=\text { const. }
$$

Then

$$
\frac{\partial \phi}{\partial u}: \frac{\partial \phi}{\partial v}: \frac{\partial \phi}{\partial w}=\frac{\partial w}{\partial u}: \frac{\partial w}{\partial v}:-1
$$


The jacobian of $x$ and $y$ with regard to $u$ and $v$ should now vanish, or

$$
\begin{gathered}
\left|\begin{array}{c}
\frac{\partial x}{\partial u}+\frac{\partial x}{\partial w} \frac{\partial w}{\partial u} \frac{\partial x}{\partial v}+\frac{\partial x}{\partial w} \frac{\partial w}{\partial v} \\
\frac{\partial y}{\partial u}+\frac{\partial y}{\partial w} \frac{\partial w}{\partial u} \frac{\partial y}{\partial v}+\frac{\partial y}{\partial w} \frac{\partial w}{\partial v}
\end{array}\right|=0, \\
\frac{\partial \phi}{\partial u} \frac{\partial(x, y)}{\partial(v, w)}+\frac{\partial \phi}{\partial v} \frac{\partial(x, y)}{\partial(w, u)}+\frac{\partial \phi}{\partial w} \frac{\partial(x, y)}{\partial(w, v)}=0, \\
\frac{\partial \phi}{\partial u} \frac{\partial\left(x, \frac{y}{y}\right)}{\partial(v, w)}+\frac{\partial \phi}{\partial v} \frac{\partial(\bar{x}, \bar{y})}{\partial(w, u)}+\frac{\partial \phi}{\partial w} \frac{\partial(x, y)}{\partial(u, v)}=0 .
\end{gathered}
$$

There will therefore exist a function of $u, v, w$ whose partial derivatives are proportional to the determinants of

$$
\left\|\begin{array}{lll}
\frac{\partial(x, y)}{\partial(v, w)} & \frac{\partial(x, y)}{\partial(w, u)} & \frac{\partial(x, y)}{\partial(u, v)} \\
\frac{\partial(\bar{x}, \bar{y})}{\partial(v, w)} & \frac{\partial(\bar{x}, \bar{y})}{\partial(w, u)} & \frac{\partial(\bar{x}, \bar{y})}{\partial(u, v)}
\end{array}\right\| .
$$

Our reasoning is reversible throughout, hence we have:

THEOREM 19. If $x$ and $y$ be functions of three real parameters, $u, v, w, a$ necessary and sufficient condition that the system so defined should contain one, and. hence, an infinite number of curves is that the Pfaff equation

$$
\left|\begin{array}{ccc}
d u & d v & d w \\
\frac{\partial(x, y)}{\partial(v, w)} & \frac{\partial(x, y)}{\partial(w, u)} & \frac{\partial(x, y)}{\partial(u, v)} \\
\frac{\partial(\bar{x}, \bar{y})}{\partial(v, w)} & \frac{\partial(\bar{x}, \bar{y})}{\partial(w, u)} & \frac{\partial(\bar{x}, \bar{y})}{\partial(u, v)}
\end{array}\right|=0
$$

should be integrable.

It is time to see what form this condition takes when the system is expressed in the form (17).

Suppose that we have a system of curves, depending on a real parameter $R$,

$$
y=y(x, R), \quad \bar{y}=\bar{y}(\bar{x}, R)
$$

which is contained wholly in the variety. We have

$$
F(x, y(x, R), \bar{x}, \bar{y}(\bar{x}, R)) \equiv 0 .
$$


We may treat $x$ and $\bar{x}$ as independent variables and differentiate;

$$
\begin{gathered}
\frac{\partial F}{\partial x}+\frac{\partial F}{\partial y} \frac{\partial y}{\partial x}=\frac{\partial F}{\partial \bar{x}}+\frac{\partial F}{\partial \bar{y}} \frac{\partial \bar{y}}{\partial \bar{x}}=0 \\
\frac{\partial^{2} F}{\partial x \partial \bar{x}}+\frac{\partial^{2} F}{\partial y \partial \bar{x}} \frac{\partial y}{\partial x}+\frac{\partial^{2} F}{\partial x \partial \bar{y}} \frac{\partial \bar{y}}{\partial \bar{x}}+\frac{\partial^{2} F}{\partial y \partial \bar{y}} \frac{\partial y}{\partial x} \frac{\partial \bar{y}}{\partial \bar{x}}=0 \\
\left|\begin{array}{lll}
\frac{\partial^{2} F}{\partial x \partial \bar{x}} & \frac{\partial^{2} F}{\partial x \partial \bar{y}} & \frac{\partial F}{\partial x} \\
\frac{\partial^{2} F}{\partial y \partial \bar{x}} & \frac{\partial^{2} F}{\partial y \partial \bar{y}} & \frac{\partial F}{\partial y} \\
\frac{\partial F}{\partial \bar{x}} & \frac{\partial F}{\partial \bar{y}} & 0
\end{array}\right|=0 \quad \text { when } F=0 .
\end{gathered}
$$

Suppose conversely that the first equation holds when the second does. The jacobian in $\bar{x}$ and $\bar{y}$ of $F$ and $\frac{\partial F}{\partial x} / \frac{\partial F}{\partial y}$ vanishes, so that $F$ can be written

$$
\phi\left(x, y, \frac{\partial F}{\partial x} / \frac{\partial F}{\partial y}\right)=0
$$

and this holds when we treat $\bar{x}$ and $\bar{y}$ as variables independent of $x$ and $y$.

If, then, we give to $\bar{x}$ and $\bar{y}$ such values that

$$
\frac{\partial F}{\partial x}=R \frac{\partial F}{\partial y}
$$

where $R$ is any real constant, we have $\infty^{1}$ curves in our variety, namely

$$
\phi(x, y, R)=0 \text {. }
$$

The invariants of a three-parameter system of points are less simple and interesting than those of a congruence. The squared distance element

$$
d x^{2}+d y^{2}
$$

is a ternary quadratic form in $d u: d v: d w$, but its discriminant is equal to zero, being

$$
\left[\frac{\partial(x, y, 0)}{\partial(u, v, w)}\right]^{2}
$$

Another invariant is the discriminant of

$$
d x d \bar{x}+d y d \bar{y}
$$


We find that this is

$$
\frac{1}{4}\left[\frac{\partial(x, y, \bar{x})}{\partial(u, v, w)} \frac{(\partial \bar{x}, \bar{y}, x)}{\partial(u, v, w)}+\frac{\partial(x, y, \bar{y})}{\partial(u, v, w)} \frac{\partial(\bar{x}, \bar{y}, y)}{\partial(u, v, w)}\right] \geqq 0 .
$$

Every three-parameter system of points will have a thread on each minimal line, as we see by counting parameters. The differential equations of these threads are obtained by setting

$$
\begin{gathered}
d x \pm i d y=d \bar{x} \pm i d \bar{y}=0, \\
\frac{d u}{H(v, w) \pm K(v, w)}=\frac{d v}{H(w, u) \pm K(w, u)}=\frac{d w}{H(u, v) \pm K(u, v)} .
\end{gathered}
$$

\section{TANGENTS}

Let us now look at the tangent chains to a three-parameter system. Let us see where chain (3) meets the system (19). Expanding in MacLaurin's series for $t$ we find

$$
\begin{gathered}
0=F(x, y, \bar{x}, \bar{y})+t\left\{\rho\left[\left(x^{\prime}-x\right) \frac{\partial F}{\partial x}+\left(y^{\prime}-y\right) \frac{\partial F}{\partial y}\right]\right. \\
\left.+\bar{\rho}\left[\left(\bar{x}^{\prime}-\bar{x}\right) \frac{\partial F}{\partial \bar{x}}+\left(\bar{y}^{\prime}-\bar{y}\right) \frac{\partial F}{\partial \bar{y}}\right]\right\}+\cdots \\
+t^{2}\left[\alpha \rho^{2}+2 \beta \rho \bar{\rho}+\bar{\alpha} \bar{\rho}^{2}\right]+\cdots .
\end{gathered}
$$

Let us assume that $(x, y)$ is a point of the variety. The equation in $t$ has one root $t=0$. If, furthermore, $\left(x^{\prime}, y^{\prime}\right)$ lies on the straight line

$$
\left(x^{\prime}-x\right) \frac{\partial F}{\partial x}+\left(y^{\prime}-y\right) \frac{\partial F}{\partial y}=0
$$

since the conjugate expression vanishes at the same time, we see that there is another root $t=0$ regardless of $\rho$, i. e., every chain from $(x, y)$ to $\left(x^{\prime}, y^{\prime}\right)$ is tangent. This line will always exist, unless we are at a singular point where all four partial derivatives vanish. We shall speak of this line as the "tangent" to the variety at the point in question. Note that it will meet the variety in a thread having a double point, or an isolated singularity, at the point of contact. If, on the other hand, $\left(x^{\prime}, y^{\prime}\right)$ does not lie on this line, there is just one value of $\rho: \bar{\rho}$ to suit.

THEOREM 20. Every point not on the tangent line to a three-parameter system at a non-singular point may be connected with that point by just one chain touching the variety there. 
THEOREM 21. The tangent to a three-parameter system at a non-singular point meets the variety in a thread having a double point or isolated singularity there. Every chain of the line through the point of contact is tangent to the variety.

If we equate both the coefficients of $t$ and of $t^{2}$ to zero, and eliminate $\rho: \bar{\rho}$ we have an equation which may be expressed in the symbolic form:

$$
\left[\alpha\left(x^{\prime}-x\right)+\beta\left(y^{\prime}-y\right)\right]^{2}\left[\bar{\alpha}\left(x^{\prime}-x\right)+\bar{\beta}\left(\bar{y}^{\prime}-\bar{y}\right)\right]^{2}=0 .
$$

This equation will surely be satisfied by the point $(x, y)$ and if it be satisfied by any other point, then the coördinates of every point of the line from there to $(x, y)$ will satisfy it. We have thus, if we have anything, a one-parameter family of lines through $(x, y)$ and the tangent will be a singular line of the family. If $\left(x^{\prime}, y^{\prime}\right)$ be a point on a line of the system, which is not the tangent, the corresponding values of $\rho$ and $\bar{\rho}$ are uniquely determined; when $\left(x^{\prime}, y^{\prime}\right)$ is on the tangent, there are two sets of values of $\rho: \bar{\rho}$, usually distinct, which fit.

THEOREM 22. If there be any point not on the tangent at a non-singular point, whose tangent chain at the non-singular point osculates the three-parameter variety there, then there are $\infty^{3}$ such points which generate a one-parameter variety of lines through the given point of the variety, the tangent being singular for the family.

Let us conclude our work by giving an important geometrical interpretation to equation (19). Let us assume that the origin is the point in which we are interested, and the $y$ axis is the tangent there. We may then express our variety in the form:

$$
\begin{aligned}
0= & a y+\bar{a} \bar{y}+\left(b x^{2}+2 c x y+d y^{2}\right)+\left(\bar{b} \bar{x}^{2}+2 \bar{c} \bar{x} \bar{y}+\bar{d} \bar{y}^{2}\right) \\
& +(B x \bar{x}+\gamma x \bar{y}+\bar{\gamma} \bar{x} y+D y \bar{y})+\cdots
\end{aligned}
$$

Suppose, now, the origin satisfies equation (19) without being a double point of the variety. We easily find

$$
B=0 \text {. }
$$

To find the nature of the thread cut by the variety in its tangent, let us put.

$$
y=0, x=X+i Y
$$

We have, in the Gauss plane,

$$
0=(B+b \bar{b}) X^{2}+2 i(b \bar{b}) x y+(B-b \bar{b}) Y^{2}+\cdots .
$$

This is a real curve (if it be anything) with the origin as a double point or isolated singularity. The tangents to the two branches will be at right angles if

$$
B=0 \text {. }
$$


If, then, we remember the definition of orthogonal chains given at the beginning of the present article, we reach the curious result:

THEOREM 23. A necessary and sufficient condition that a three-parameter family of points should contain a one-parameter family of curves is that tangent chains to the two branches of the thread cut by a tangent to the variety should be mutually orthogonal at the point of contact.

HARVARD UNIVERSTTY,

Cambridge, mass. 